\title{
Structure and properties of coralline red alga: from helical configuration to alternating layers
}

\author{
Nuphar Bianco-Stein ${ }^{1}$, Iryna Polishchuk ${ }^{1}$, Paul Zaslansky ${ }^{2}$, Alexander Katsman ${ }^{1}$ and Boaz Pokroy ${ }^{1 *}$ \\ ${ }^{1}$ Department of Materials Science and Engineering and the Russell Berrie Nanotechnology Institute, Technion-Israel Institute of \\ Technology, 32000 Haifa, Israel. \\ ${ }^{2}$ Department of Restorative and Preventive Dentistry, Institute for Dental and Craniofacial Sciences, Charité-Universitätsmedizin \\ Berlin, 14197 Berlin, Germany. \\ bpokroy@technion.ac.il.
}

Through controlled biomineralization, organisms yield complicated structures with specific functions. Here, Jania sp., an articulated coralline red alga that secretes high-Mg calcite as part of its skeleton, is in focus. It is shown that Jania sp. exhibits a remarkable structure, which is highly porous (with porosity as high as $64 \mathrm{vol} \%$ ) and reveals several hierarchical orders from the nano to the macroscale. It is shown that the structure is helical, and proven that its helical configuration provides the alga with superior compliance that allows it to adapt to stresses in its natural environment. Thus, the combination of high porosity and a helical configuration result in a sophisticated, light-weight, compliant structure [1]. Very recently, we also showed that the high-Mg calcite cell wall nanocrystals of Jania sp. are arranged in layers with alternating Mg contents. Such non-homogenous elemental distribution assists the alga in preventing fracture caused by crack propagation. We further discover that each one of the cell wall nanocrystals in Jania sp. is not a single crystal as was previously thought, but rather comprises Mg-rich calcite nanoparticles demonstrating various crystallographic orientations, arranged periodically within the layered structure [2]. We also show that these Mg-rich nanoparticles are present in yet another species of the coralline red algae, Corallina sp., pointing to the generality of this phenomenon. To the best of our knowledge this is a first report on the existence of Mg-rich nanoparticles in the coralline red algae mineralized tissue. We envisage that our findings on the bio-strategy found in the alga to enhance the fracture toughness will have an impact on the design of structures with superior mechanical properties.

[1] Bianco-Stein N, Polishchuk I, Seiden G, Villanova J, Rack A, Zaslansky P and Pokroy B. Helical Microstructures of the Mineralized Coralline Red Algae Determine Their Mechanical Properties. Adv Sci 2020; 7:2000108.

[2] Bianco-Stein N, Polishchuk I, Lang A, Atiya G, Villanova J, Zaslansky P, Katsman A and Pokroy B. Structural and Chemical Variations in the Calcitic Segments of Coralline Red Algae Lead to Improved Crack Resistance. Acta Biomater 2021; DOI:10.1016/j.actbio.2021.05.040.

Keywords: biomineralization, red alga, Mg-calcite 